\title{
Cell-Based No-Wash Fluorescence Assays for Compound Screens Using a Fluorescence Cytometry Plate Reader ${ }^{\text {s }}$
}

\author{
○ Kirill Gorshkov, Manisha Pradhan, Miao Xu, Shu Yang, Emily M. Lee, Catherine Z. Chen, \\ Min Shen, and $\odot$ Wei Zheng
}

National Center for Advancing Translational Sciences, National Institutes of Health, Rockville, Maryland

Received February 3, 2020; accepted June 1, 2020

\begin{abstract}
High-throughput cell-based fluorescent imaging assays often require removal of background fluorescent signal to obtain robust measurements. Processing high-density microplates to remove background signal is challenging because of equipment requirements and increasing variation after multiple plate wash steps. Here, we present the development of a wash-free cellbased fluorescence assay method for high-throughput screening using a laser scanning fluorescence plate cytometer. The cytometry data consisted of cell count and fluorescent intensity measurements for phenotypic screening. We obtained robust screening results by applying this assay methodology to the lysosomal storage disease Niemann-Pick disease type A. We further demonstrated that this cytometry method can be applied to the detection of cholesterol in Niemann-Pick disease type C. Lastly, we used the Mirrorball method to obtain preliminary results for the detection of Zika and Dengue viral envelope protein. The advantages of this assay format include 1) no plate washing, 2) 4-fold faster plate scan and analysis time, 3)
\end{abstract}

high throughput, and 4) $>10$-fold smaller direct data files. In contrast, traditional imaging assays require multiple plate washes to remove the background signal, long plate scan and data analysis times, and large data files. Therefore, this versatile and broadly applicable Mirrorball-based method greatly improves the throughput and data quality of imagebased screening by increasing sensitivity and efficiency while reducing assay artifacts.

\section{SIGNIFICANCE STATEMENT}

This work has resulted in the development of broadly applicable cell-based fluorescence imaging assays without the requirement of washing out reagents to reduce background signal, which effectively decreases the need for extensive plate processing by the researcher. We demonstrate this high-throughput method for drug screening against lysosomal storage diseases and a commonly used viral titer assay.

\section{Introduction}

Phenotypic screening is an invaluable and unbiased tool for high-throughput drug discovery and development (Priest and Erdemli, 2014). Phenotypic screens, as opposed to targetbased screens, determine the effect of a drug candidate on relevant functions or disease phenotypes in cells or model organisms. Fluorescence microscopy provides a rich amount of information within each image, and high-content analysis provides a cornucopia of information about drug potency and

This work was supported by the Intramural Research Programs of the National Center for Advancing Translational Sciences, National Institute of Health (W.Z.)

The authors report no conflicts of interest.

Portions of this work were previously presented: Gorshkov, Kirill et al. "High-Throughput Assay Development for Niemann-Pick Disease Type A Small Molecule Therapeutics." Molecular genetics and metabolism. 123.2 (2018): n. pag. Web. June 19-24, Proctor Academy. Andover, NH. Gordon Research Conference Lysosomes and Endocytosis

https://doi.org/10.1124/jpet.120.265207.

S This article has supplemental material available at jpet.aspetjournals.org. efficacy (Zanella et al., 2010). The advent of automated highcontent microscopes allows researchers to generate tremendous amounts of data, often more than can be reasonably analyzed without incorporating sophisticated machine learning algorithms. The process is often challenging because highquality imaging samples are needed to obtain accurate results with high fidelity. In many cases, an even more challenging step in deriving conclusions from high-content screening is image analysis and data processing because of the well-to-well variations and diverse cell morphology.

Sample preparation for fluorescence microscopy typically requires significant amounts of user input to produce images with high signal-to-background ratios and low noise. Effective removal of background signal is essential for high-content microscopy, as software-assisted object identification and segmentation are often dependent on the fluorescence intensity of the object and its background area. Low signal-tobackground ratios prevent accurate object segmentation. Therefore, the data may not be representative of single cells, or in some cases, entire cell areas may not be identified,

ABBREVIATIONS: BODIPY-SM, BODIPY-sphingomyelin; DENV, Dengue virus; DMEM, Dulbecco's modified Eagle's medium; FDA, Food and Drug Administration; GSK3- $\beta$, glycogen synthase kinase $3-\beta$; HP $\beta C D$, hydroxypropyl- $\beta$-cyclodextrin; HTS, high-throughput screening; iPSC, induced pluripotent stem cell; LC3, microtubule-associated proteins 1A/1B light chain 3B; LSD, lysosomal storage disease; M $\beta C D$, methyl- $\beta$-cyclodextrin; mTOR, mammalian target of rapamycin; NPA, Niemann-Pick disease type A; NPC, Niemann-Pick disease type C; NSC, neural stem cell; PDGFR, platelet-derived growth factor receptor; PFO, perfringolysin O*; PKA, protein kinase A; WT, wild-type; ZIKV, Zika virus. 
leading to improper estimates of whole-cell or organelle-level intensity, areas, and object counts, among other critical highcontent parameters. New laser-based scanning imaging technologies are now available to overcome such hurdles to develop high-quality cell-based fluorescence assays with rapid plate reading and simplified on-site data analysis for drug discovery (England et al., 2015).

The Mirrorball laser scanning fluorescent plate cytometer enables researchers to conduct washless cell-based fluorescence assays with high-density microplates for high-throughput drug screening, which has been used in the bead-based detection for antibody screening (Rasmussen et al., 2015; Balmaseda et al., 2017; Nishiyama et al., 2017). In this study, we first applied this method to cells from patients with lysosomal storage disease (LSD) that have a substrate accumulation phenotype. In the case of Niemann-Pick disease type A (NPA), a fatal childhood developmental disorder characterized by a lack of acid sphingomyelinase encoded by the sphingomyelin phosphodiesterase 1 gene, the substrate sphingomyelin accumulates in the lysosome (Schuchman and Desnick, 2017). We tested the capability of the Mirrorball to detect cellular accumulation of substrate using wild-type and NPA human skin fibroblasts. Assay parameters were optimized for 384well microplates, and a pilot screen identified several compounds that can reduce the intracellular concentration of 1,3,5,7-Tetramethyl-8-phenyl-4,4-difluoroboradiazaindacene (BODIPY)-sphingomyelin (BODIPY-SM), a green fluorescent substrate that accumulates in the lysosome of NPA cells. In addition, we employed a fluorescent dye-conjugated protein, perfringolysin $\mathrm{O}^{*}(\mathrm{PFO})$, to detect cholesterol accumulation in Niemann-Pick disease type C (NPC) neural stem cells (NSCs) (Maekawa et al., 2016; Johnson et al., 2017). Lastly, we applied this methodology to no-wash flaviral titer assays. Altogether, our newly developed methods are broadly applicable as primary screening tools to robustly identify hits for drug discovery and development campaigns.

\section{Methods}

Reagents. Ninety-six-well tissue culture plates (655090) and 384-well tissue culture plates (781096) were purchased from Greiner Bio-One (Monroe, NC). The 1536-well plates (E8 style) were purchased from Aurora Microplates (Whitefish, MT). DMEM (11965-092), phenol red-free DMEM (21063029), PenStrep (15070-063), knockout DMEM/F12 (12660012), StemPro Neural Supplement (A1050801), basic fibroblast growth factor (PHG0024), epidermal growth factor (PHG0314), Glutamax (35050061), Dulbecco's phosphate buffered saline (14190), recombinant human vitronectin (A14700), BODIPY-C12 sphingomyelin (D7711), LysoTracker Deep Red (L12492), and 1, 5bis $\{[2$-( di- methylamino) ethyl] amino $\}$ - 4, 8- dihydroxyanthracene-9, 10-dione (DRAQ5) (62252) and Live-Cell Imaging Solution (A14291DJ) were purchased from ThermoFisher Scientific (Waltham, MA). Matrigel (354234) was purchased from Corning (Corning, NY). Methyl cellulose (M0512) was purchased from Millipore Sigma (St. Louis, MO). $\delta$-Tocopherol (T2028) was purchased from Sigma-Aldrich and purified by high-performance liquid chromatography. Mirsky's Fixative (15560) and paraformaldehyde (15714-S) were purchased from Electron Microscopy Sciences (Hatfield, PA). Cell Staining Buffer (420201) was purchased from BioLegend (San Diego, CA). Nuclear Green LCS1 (17540) was purchased from AAT Bioquest. M $\beta$ CD was purchased from Carbosynth (OD29274, 2,6-dimethyl-b-cyclodextrin). Hydroxypropyl- $\beta$-cyclodextrin (HP $\beta$ CD) was purchased from Millipore (number 15340, hydroxypropyl- $\beta$-cyclodextrin). iFluor-647-PFO* (CB-170411c) was purchased from Codex Biosolutions (Gaithersburg,
MD) and labeled by AAT Bioquest (Sunnyvale, CA). Wild-type (WT) (male; GM05659), NPC1 (female; GM03123), and NPA (Female; GM13205) fibroblasts were purchased from Coriell Institute for Medical Research (Camden, NJ). NPC1 NSCs were differentiated from human patient fibroblast-derived induced pluripotent stem cells (iPSCs) as described previously (Yu et al., 2014). Vero cells (American Type Culture Collection[ATCC] CCL-81) and Aedes albopictus clone C6/36 cells (ATCC CRL-1660) for PRVABC59-ZIKV strain stocks were obtained from ATCC (Manassas, VA). Zika virus (ZIKV) and Dengue virus (DENV) were gifts from Dr. Hengli Tang at Florida State University.

Cell Culture. Fibroblasts were cultured in DMEM with 10\% FBS and $5 \%$ penicillin/streptomycin. Cells were plated at a density of 500 cells per well per $15 \mu \mathrm{l}$ of phenol red-free DMEM complete with FBS and penicillin/streptomycin into 384-well plates, unless otherwise indicated, with the Multidrop Combi Liquid Dispenser. After 4 hours, $15 \mu \mathrm{l}$ of a 1:2500 dilution of stock BODIPY-C12 sphingomyelin in complete DMEM phenol red-free media was added to cells. After 24 hours, compounds were added to the plates using Multidrop or a PinTool (Wako Automation, San Diego, CA), and cells were incubated for an additional 72 hours.

Patient iPSC-derived neural stem cells (NPC1 NSCs) were cultured at 1000 cells per well per $4.0 \mu$ l of neural stem cell media consisting of knockout DMEM/F12, fibroblast growth factor beta, epidermal growth factor, StemPro Supplement, and Glutamax, $50 \mu \mathrm{g} / \mathrm{ml}$ of recombinat human vitronectin. After 24 hours, cells were treated with an additional $2.0 \mu \mathrm{l}$ of $7.5 \% \mathrm{FBS}$ for a final concentration of $2.5 \%$ FBS to induce accumulation of cholesterol. After 2 hours, cells were treated with cyclodextrin using the Wako PinTool for an additional 72 hours.

ZIKV and DENV Culture. Zika virus or Dengue virus stocks were generated in A. albopictus clone C6/36 cells from PRVABC59ZIKV strain or Dengue virus type $2-16681$ stocks. To amplify viral stocks, a T-75 flask of C6/36 cells (90\%-95\% confluency) was inoculated with 1e6 Zika virions in low volume $(3 \mathrm{ml})$ for 1 hour, with rocking to disperse media every 15 minutes. After 1 hour, $17 \mathrm{ml}$ of media was added, and $\mathrm{C} 6 / 36$ cells were maintained at $28^{\circ} \mathrm{C}$ in $5 \% \mathrm{CO}_{2}$ for 7 days. At day 7 and day 8 post-viral inoculation, supernatants were harvested, filtered, and stored at $-80^{\circ} \mathrm{C}$. ZIKV and DENV titer was determined by focus forming unit assay.

ZIKV and DENV Titer Assay. Briefly, Vero cells were seeded overnight into 96-well plates to achieve a confluent monolayer the next day. ZIKV or DENV was then added to the naïve Vero cell plate after removal of maintenance media in triplicate, 10-fold serial dilutions. After 2 hours of incubation, the viral inoculum was removed, methyl cellulose overlay (equal parts $2 \times$ DMEM maintenance media and $2 \%$ methyl cellulose solution) was added to each well, and cells were incubated for 48 hours at $37^{\circ} \mathrm{C}$. The methyl cellulose overlay was then removed by aspiration, and wells were fixed with $100 \mu l 4 \%$ paraformaldehyde for 15 minutes. Fixing solution was removed by BlueWasher (Blue Cat Bio), and cells were permeabilized with $0.5 \%$ PBS containing $0.5 \%$ Triton X-100 for 10 minutes. Permeabilization buffer was removed by BlueWasher, and cells were then blocked with $50 \mu \mathrm{l}$ PBS containing $0.1 \%$ Triton X-100, $10 \%$ normal goat serum, and $1 \%$ bovine serum albumin for 1 hour at room temperature while rocking before addition of $50 \mu$ l Alexa Fluor 488-conjugated primary antibody solution (Clone 4G2, conjugated with Alexa Fluor 488, 1:250 in PBS containing $0.1 \%$ Triton X-100, $10 \%$ normal goat serum, and $1 \%$ bovine serum albumin for a final concentration of 1:500) for 2 hours at room temperature while rocking (Yang et al., 2018). Plates were then imaged on the Mirrorball (TTP Labtech). The ZIKV and DENV titer assay was conducted according to a preset plan as a proof of concept using three wells per condition in a 96-well plate.

Dye Staining. NPA fibroblasts were treated with $50 \mathrm{nM}$ LysoTracker Deep Red and $1 \mu$ M DRAQ5 for 1 hour in Live-Cell Imaging Solution directly into the culture plates. Then, cells were fixed in $1 \times$ Mirsky's Fixative for an additional 1 hour.

NPC NSCs were first incubated with $1 \mu \mathrm{M}$ Nuclear Green LCS1 to stain the nuclei for 15 minutes. Then, the cells were fixed, permeabilized, 
and blocked for 30 minutes. Media were evacuated using a BlueWasher (BlueCatBio) before staining with $4 \mu \mathrm{g} / \mathrm{ml}$ iFluor647-PFO for 1 hour in blocking buffer.

Cell Imaging. NPA fibroblasts in 384-well plates after staining were loaded into the Mirrorball (TTP Labtech) and scanned with the indicated optimization settings, including laser power, photomultiplier tube (PMT) voltage, and acquisition triggers. For scanning of whole 384well plates, cells were imaged at the default laser power of $6 \mathrm{~mA}$, FL2 (green fluorescence) PMT voltage of $400 \mathrm{~V}$, FL4 (red fluorescence) PMT voltage of $500 \mathrm{~V}$, and red acquisition triggering with a sensitivity of 2 S.D. above background. Scan lines were set to a resolution of $4 \mu \mathrm{m}$. Median (mean FL2 intensity) and object count using the FL4 channel were the object classification parameters used to report the accumulation of BODIPY-SM and cell number, respectively.

For scanning NPC1 fibroblasts after dye staining in 1536-well plates, we used a 488-nm laser power of $2 \mathrm{~mA}$ and $640-\mathrm{nm}$ laser power of $3 \mathrm{~mA}$, FL2 PMT voltage of $350 \mathrm{~V}$, FL4 PMT voltage of $400 \mathrm{~V}$, and green acquisition triggering with a sensitivity of 3 S.D. above background. Scan lines were set to a resolution of $3 \mu \mathrm{m}$. Other settings included minimum feature length of $1 \mu \mathrm{m}$, max feature length of $100 \mu \mathrm{m}$, feature extension of $2 \mu \mathrm{m}$, and x-y separation of $1 \mu \mathrm{m}$. Median (mean FL4 intensity) and object count using the FL2 channel were the object classification parameters used to report the accumulation of cholesterol and cell number, respectively. The lysosomal storage disease assays using BODIPY-SM and PFO staining were conducted according to a preset plan using three independent experiments. Assay optimization included many technical replicate wells. Follow-up screening on selected compounds was done using three independent experiments with three technical replicate plates per experiment.

Images were also taken on the IN Cell 2200 for comparison (GE Healthcare).

Statistical Analysis. All assays were evaluated without blinding. Raw data were exported from the Mirrorball's Cellista software (TTP Labtech), loaded into Microsoft Excel, and processed using standard statistical methods in Graphpad Prism version 7.04. The median (mean FL2 intensity) can be described as the median value of the mean FL2 intensity of all the objects in the well. Each object's mean FL2 intensity is recorded, and the median of that distribution is reported for each well. Then, the average of all the wells for that condition is taken as a final measurement. The data in Fig. 3B were normalized to the lowest median (mean FL2 intensity) of treated cells ( $\delta$-tocopherol $+150 \mathrm{mM} \mathrm{HP} \beta \mathrm{CD}$ condition; $100 \%$ efficacy) and the median (mean FL2 intensity) of control cells treated with DMSO ( $0 \%$ efficacy). For NPA screening, data were normalized to WT cells as a positive control (100\% efficacy) and NPA cells as a negative control (0\% efficacy). For NPC1 NSCs, data were normalized to NPC1 without FBS (100\% efficacy) and NPC1 cells with FBS (0\% efficacy). Images were processed using Fiji version 1.51 (National Institutes of Health).

\section{Results}

Initial Assay Optimization. We first set out to optimize the green fluorescent substrate concentration and the red fluorescent counterstain using the NPA fibroblasts. The Mirrorball has a five-channel photomultiplier tube array to capture information from near-UV to near-infrared fluorescent signals. In this work, we used the FL2 green channel and FL4 red channel for BODIPY-SM and LysoTracker Deep Red dye combined with the DRAQ5 nuclear dye, respectively. Increased fluorescence staining signals of BODIPY-SM and LysoTracker indicated the accumulated sphingomyelin lipids and enlarged lysosomes in cells, whereas DRAQ5 staining outlined the nuclear structure. The laser scanning technology coupled with background subtraction software provided an opportunity to view the images in raw TIFF as well as background-subtracted object data (Fig. 1A). Further, the Cellista software allowed for viewing of the object outlines to assess the proper segmentation of cell object data (Fig. 1B). The various parameter profiles could be viewed as a histogram of object-level data. We tested four different concentrations of BODIPY-SM and LysoTracker to identify nonsaturating conditions to be within the detection limit of the system. In this assay, the BODIPY-SM fluorescence in the green FL2 channel was the primary signal to be measured. A concentration of 460 nM BODIPY-SM produced a high saturating peak intensity, whereas $230 \mathrm{nM}$ BODIPY-SM was less saturating, with most of the objects exhibiting a peak green intensity between 4000 and 10,000 a.u. A concentration of $58.0 \mathrm{nM}$ BODIPY-SM had a low peak intensity signal (Fig. 1C). Similarly, $50.0 \mathrm{nM}$ LysoTracker produced an appropriate range of red peak intensities. One useful feature of the Mirrorball is the ability to view the threedimensional object profile to see the fluorescent topology and baseline intensity value. WT fibroblasts exhibit lower intensities than NPA fibroblasts in the FL2 green channel (BODIPYSM) (Fig. 1D). In summary, our initial optimization assays determined the optimal concentration of BODIPY-SM to be $230 \mathrm{nM}$ with $50 \mathrm{nM}$ LysoTracker Deep Red.

Optimizing Cell Density. Next, we optimized cell density for WT and NPA fibroblasts. Five hundred cells per well in a 384-well plate produced a confluent culture 96 hours after seeding cells, whereas 250 and 125 cells per well were subconfluent (Fig. 2A). We tested the four concentrations of BODIPY-SM with each cell density and found that each concentration produced an equal assay window for each respective density, with 500 cells per well providing the largest window of approximately 2-fold difference between WT and NPA fibroblast FL2 intensity in a 384-well plate (Fig. 2, B-D). In addition, we analyzed the object count and cell area with the four concentrations of BODIPY-SM and found that the object count, or cell number, was consistent at 500 cells per well when comparing the two cell lines (Fig. 2E). We also calculated cell area and found low variability in object area with 500 cells per well at each concentration of BODIPY$\mathrm{SM}$ (Fig. 2F). Therefore, we used 500 cells per well and $230 \mathrm{nM}$ BODIPY-SM for the next step of LysoTracker optimization.

The same cells after plate washing were then analyzed on the IN Cell Analyzer 2200 automated imaging system (Supplemental Fig. 1). The data were comparable to that obtained from the Mirrorball instrument, but the signal-tobasal ratios comparing WT with NPA green intensities at all concentrations of BODIPY-SM were reduced (Supplemental Fig. 1, A and B). Cell counts were also reduced given the single field acquisition at $20 \times$ magnification as compared with the whole-well laser scan of the Mirrorball (Supplemental Fig. 1C). Decreasing concentrations of BODIPY-SM produced concentration-dependent decreases in BODIPY-SM intensity like that observed on the Mirrorball (Supplemental Fig. 1, D and E). Importantly, the time to image the one field of the 384well plate on the IN Cell Analyzer 2200 was approximately 1 hour and 30 minutes compared with the 12-minute scan time for the Mirrorball. The Mirrorball instrument produced similar or better results compared with the traditional highthroughput imaging instrument, in addition to zero wash steps and a 7-fold faster scan time.

To further compare the two methods, we imaged an unwashed plate on the IN Cell 2200 and the same plate on the Mirrorball (Supplemental Fig. 2). We included the assay 

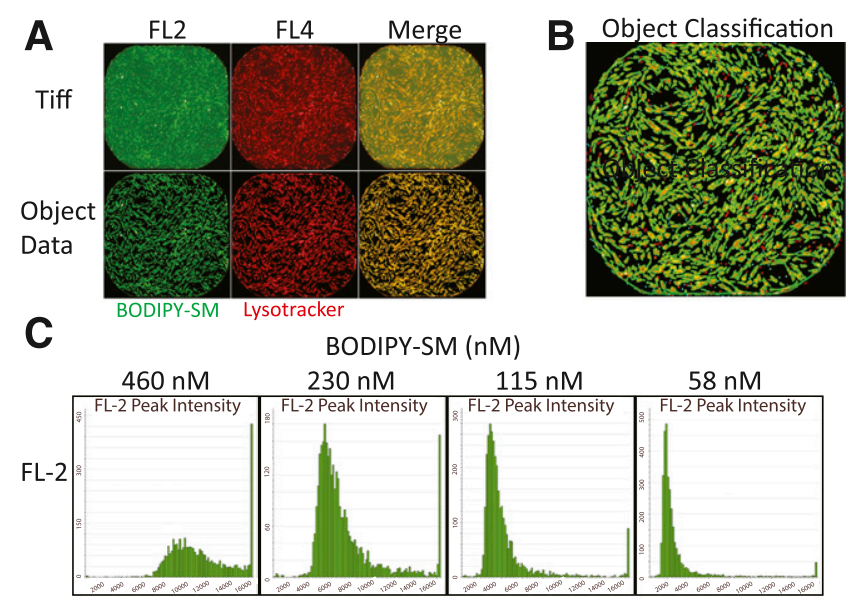

LysoTracker Deep Red (nM)

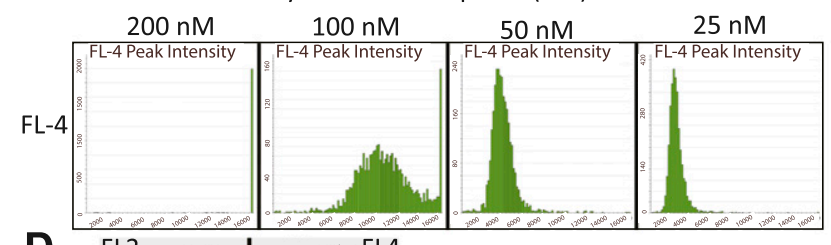

D

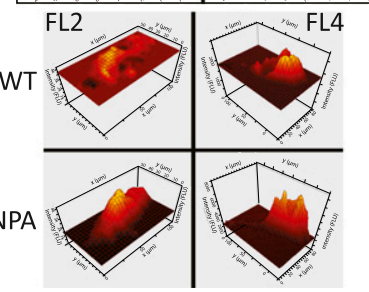

Fig. 1. Assay optimization for BODIPY-SM and LysoTracker Deep Red concentrations. (A) Raw TIFF and object data images of an entire well in the FL2 Green, FL4 Red, and merged fluorescence channels. (B) Image of the well from (A) with segmented objects. Green outlines are included cells, and red outlines are excluded cells. (C) Histograms of the number of objects $(y$-axis) and their fluorescence intensity ( $x$-axis) distribution for BODIPY-SM FL2 Green fluorescence and LysoTracker Deep Red FL4 Red fluorescence. (D) Topological representation of a WT and NPA cell in the FL2 and FL4 channel. $x$-Axis and $y$-axis are measured in micrometers, and $z$-axis fluorescence intensity is measured as fluorescence light unit (FLU).

protocol for a traditional imaging assay and the Mirrorball assay in Supplemental Table 1 that indicates significant time savings using the Mirrorball.

Validating the LysoTracker Counterstain Concentration. LysoTracker dye stains acidic organelles, including late endosomes and lysosomes. The LysoTracker dye staining increases significantly in cells from patients with NPA because lysosome sizes are enlarged because of the lipid accumulation (Long et al., 2016). Together with BODIPYSM, LysoTracker dye staining shows the localization of lysosomes in cells. In this assay, LysoTracker, along with DRAQ5, was used to fill the nucleus and cell body in the FL4 red channel for cellular segmentation. Using the established conditions for cell density and BODIPY-SM, we then confirmed that a $50 \mathrm{nM}$ LysoTracker concentration produced an acceptable nonsaturated red intensity readout (Supplemental Fig. 3A). Different LysoTracker concentrations did not affect the approximately 2-fold change of substrate accumulation between WT and NPA fibroblasts (Supplemental Fig. 3, B and $\mathrm{C})$.

Plate Statistics. Lastly, we tested the assay system for robust plate statistics (Supplemental Fig. 4). Three replicates of DMSO-treated control 384-well plates produced Z-factors of $0.54,0.53$, and 0.46 , respectively, whereas the signal-to basal ratios were 2.5-, 2.7-, and 2.5-fold. With low coefficients of variation, acceptable assay window, and Z-factor, this assay was ready for high-throughput screening.

Concentration-Responses of Positive Control Compound $\delta$-Tocopherol and Hydroxypropyl- $\beta$-Cyclodextrin. Our next goal was to determine concentration responses of positive control compound combination $\delta$-tocopherol and HP $\beta C D$ on the reduction of BODIPY-SM accumulation in NPA cells (Fig. 3). We tested five concentrations of $\delta$-tocopherol alone or in combination with $150 \mu \mathrm{M} \mathrm{HP} \beta \mathrm{CD}$. Although $\delta$-tocopherol concentrations ranging from 2.5 to $40 \mu \mathrm{M}$ dose-dependently reduced BODIPY-SM FL2 intensity, the combination with $\mathrm{HP} \beta \mathrm{CD}$ was more efficacious (Fig. 3 , A and B). There was no significant decrease in cell counts at these concentrations (Fig. 3, C and D). The data were normalized to the fluorescence intensity from cells treated with $\delta$-tocopherol in combination with $150 \mu \mathrm{M}$ HP $\beta$ CD (100\% activity) and DMSO alone (0\% activity).

We attempted to optimize these treatment conditions in a higher-throughput 1536-well plate format but found the fold difference of green intensity to be lower than that in the 384well plate format (Supplemental Fig. 5). Furthermore, there was greater variability in the efficacy of treatment and cell counts. Thus, this assay was used for further compound screening in the 384-well plate format.

Pilot Screening of the LOPAC ${ }^{1280}$ Library Using BODIPY-SM Staining in Fibroblasts Derived from Patients with NPA. We then screened the Library of Pharmacologically Active Compounds (LOPAC) library, consisting of 1280 annotated bioactive and Food and Drug Administration (FDA)-approved compounds, using the optimized 384-well plate assay conditions outlined above (Supplemental Fig. 6). LOPAC ${ }^{1280}$ is commercially available and was obtained from Sigma (LO1280). It contains pharmacologically active compounds, including modulators for neurotransmitters, phosphorylation, and ion channels, among others. From our single-concentration primary screen (Fig. 4A), we identified 64 hit compounds with an efficacy greater than $30 \%$ reduction of BODIPY-SM staining and cell count reduction of no more than $5 \%$. These hits were later used for a follow-up screen with 11 concentrations ranging from 7700 to $0.13 \mathrm{nM}$ (1-3 dilution). Of these compounds, 17 exhibited maximum responses of at least $38 \%$ with an average $\mathrm{EC}_{50}$ value of $6.5 \mu \mathrm{M}$ and average maximum response of $73 \%$ (Fig. 4B). They clustered into groups that included glycogen synthase kinase $3-\beta$ (GSK3- $\beta$ ) inhibitors, 3 '-cAMP modulators, calcium modulators, antiparasitics, and receptor tyrosine kinase inhibitors (Fig. 4, C-F). Singletons included compounds that are antioxidants, a protein kinase $\mathrm{C}$ inhibitor, a 5-hydroxy-tryptamine receptor inhibitor, a nitric oxide synthetase inhibitor, and an adenosine 3A receptor agonist (Supplemental Fig. 7; Table 1). Several of the compounds, including papaverine, dipyridamole, sunitinib, and niclosamide, are FDA-approved therapeutics. The reported $C_{\max }$ from clinical trials for these compounds is in most cases at least an order of magnitude higher than our observed $\mathrm{EC}_{50}$ values, indicating potential therapeutic uses of these compounds (Table 1 ). The results demonstrated the utility of this Mirrorball assay for HTS to identify useful compounds for further studies. 


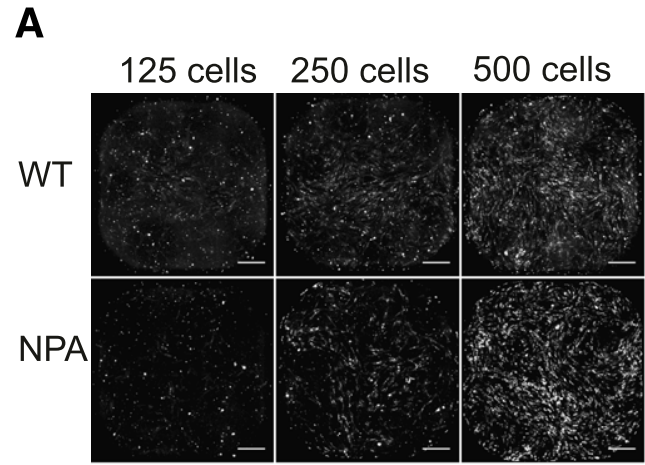

FL2 fluorescence

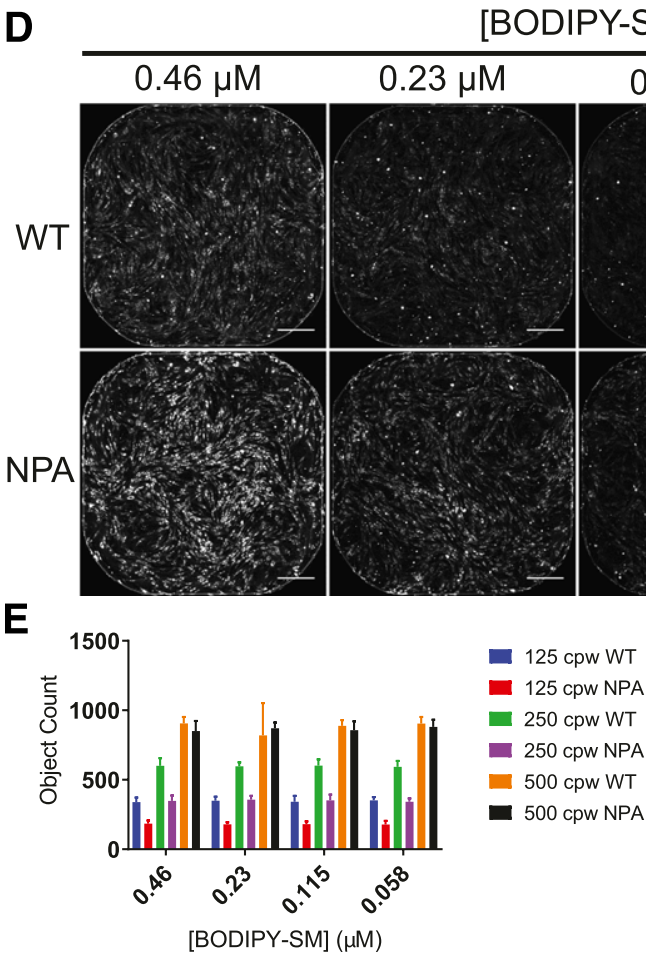

Cholesterol Detection in NPC1 NSCs with Perfringolysin O* Using the Mirrorball. We expanded the application of the newly developed method to another lysosomal storage disease, NPC1, using a dye-conjugated PFO protein to detect cholesterol accumulation, the characteristic phenotype observed in NPC1 disease cells. PFO is a secreted protein derived from the bacterium Clostridium perfringes that can selectively bind to unesterified cholesterol inside of mammalian cells (Maekawa et al., 2016; Johnson et al., 2017). Conjugated iFluor-647-PFO was used in combination with Nuclear Green LCS1 to detect cholesterol in NPC1 NSCs derived from patient iPSCs (Yu et al., 2014) (Fig. 5A). In this assay, we combined the fixation, permeabilization, and blocking into sequential additions and only a single evacuation prior to addition of the iFluor-647-PFO, after which no wash was required. We successfully miniaturized this assay to 1536 -well plates. NPC1 cells treated with $2.5 \%$ FBS (containing cholesterol) for 3 days exhibited approximately 3 -fold increased cholesterol accumulation than non-FBS-treated cells (Fig. 5, B and C). NPC1 NSCs do not accumulate cholesterol in the absence of FBS in the growth media. Furthermore, cells grown in the presence of FBS treated with the known cholesterol-reducing compounds $\mathrm{M} \beta \mathrm{CD}$ and $\mathrm{HP} \beta \mathrm{CD}$ exhibited a concentration-dependent decrease in cholesterol accumulation (Fig. 5, D and E). Both known compounds potently reduced cholesterol accumulation in NPC1 NSCs grown in the presence of FBS. M $\beta C D$ exhibited more efficacy than $\mathrm{HP} \beta \mathrm{CD}$, with $\mathrm{EC}_{50}$ values of 3.13 and $8.17 \mu \mathrm{M}$, respectively. There was no detectable toxicity at these concentrations of cyclodextrin. Thus, in addition to lipid-dye conjugates, the Mirrorball assay can also be used to effectively detect the PFO protein-dye conjugates, similar to fluorescently conjugated antibodies, in minimal-wash assay format for determination of drug potency and efficacy.

To evaluate the performance of the Mirrorball using a traditional wash protocol, prior to washing out the $\mathrm{PFO}^{*}$, 
A

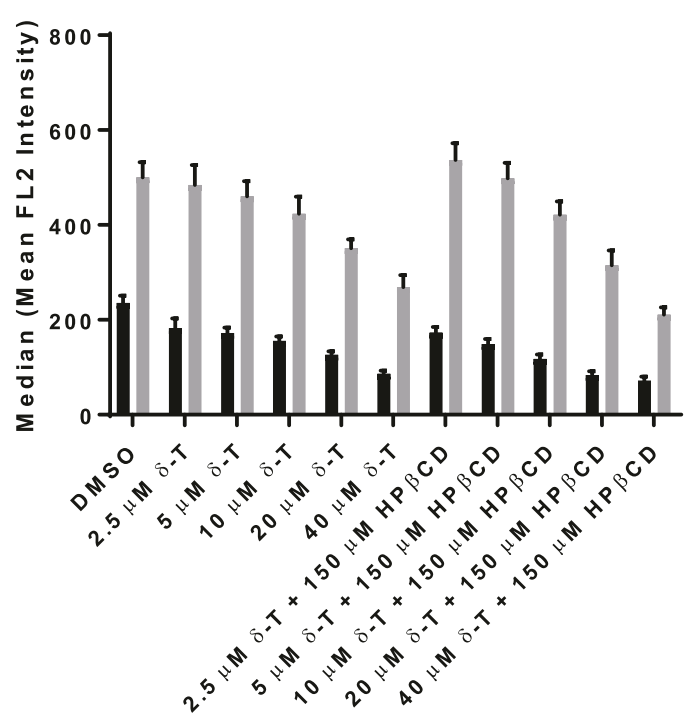

B

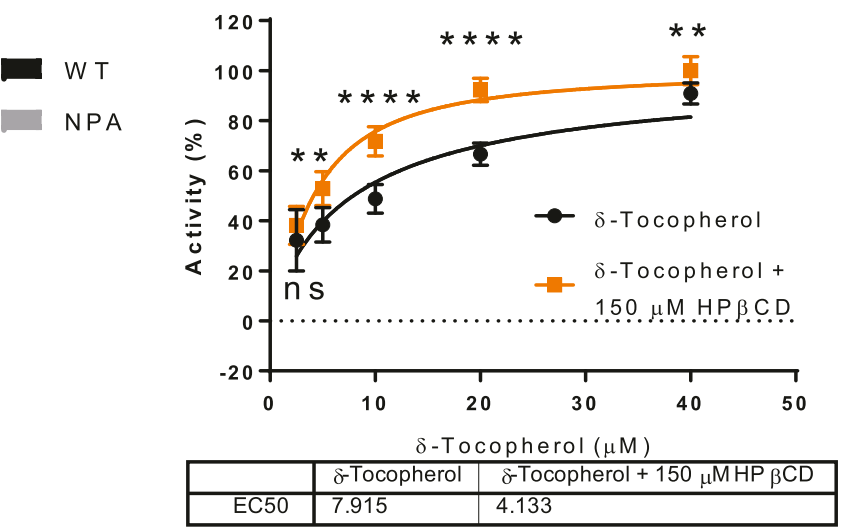

NPA

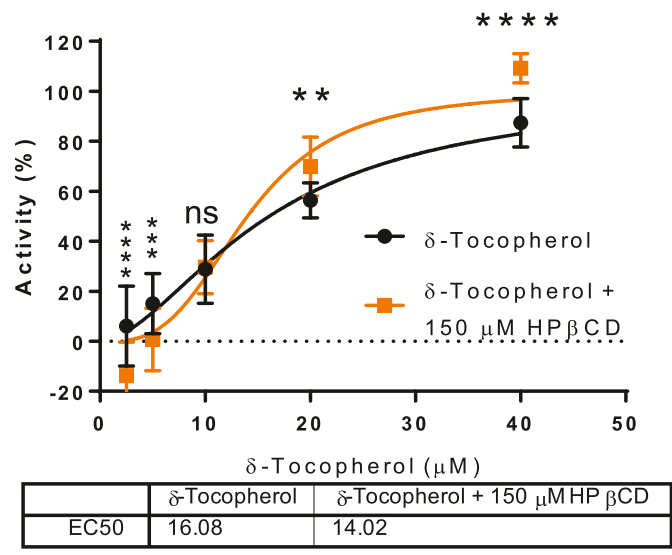

C

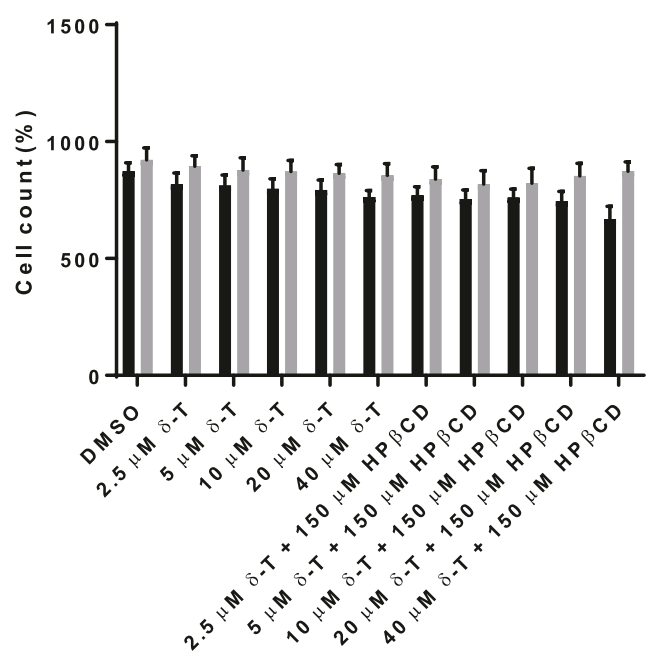

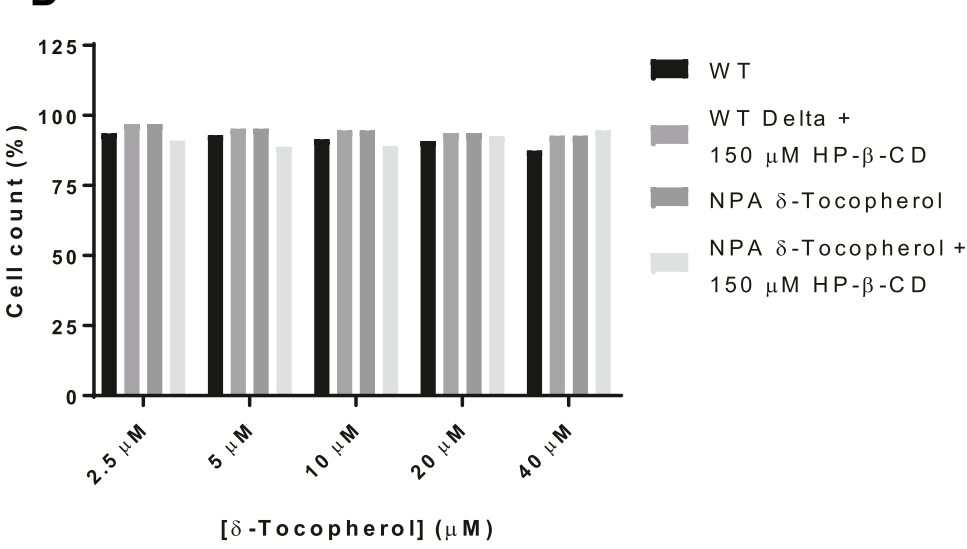

Fig. 3. Evaluation of established assay conditions using $\delta$-tocopherol combined with hydroxypropyl- $\beta$-cyclodextrin. (A) Median (mean FL2 intensity) of NPA fibroblasts treated with different concentrations of $\delta$-tocopherol combined with $150 \mu \mathrm{M} H \mathrm{HPCD}(n \geq 16$ wells). (B) $\delta$-Tocopherol in combination with $150 \mu \mathrm{M} \mathrm{HP} \beta \mathrm{CD}$ concentration-response curves from (A) for WT and NPA fibroblasts. $\mathrm{EC}_{50}$ shown below the graph. Data normalized to the lowest median (mean FL2 intensity) of treated cells ( $\delta$-tocopherol $+150 \mathrm{mM} \mathrm{HP} \beta \mathrm{CD}$ condition; 100\% efficacy) and the median (mean FL2 intensity) of control cells treated with DMSO (0\% efficacy). ${ }^{* *} P<0.01$; $* * * P<0.0001$; ns, not significant; two-way ANOVA with Sidak's multiple comparison test. (C) Cell count for treated cells in (A). (D) Normalized cell count from (C). Scale bar, $100 \mu \mathrm{m}$. Curves represent nonlinear regression curve fit with variable slope. $\delta$-T - delta-tocopherol. 
A

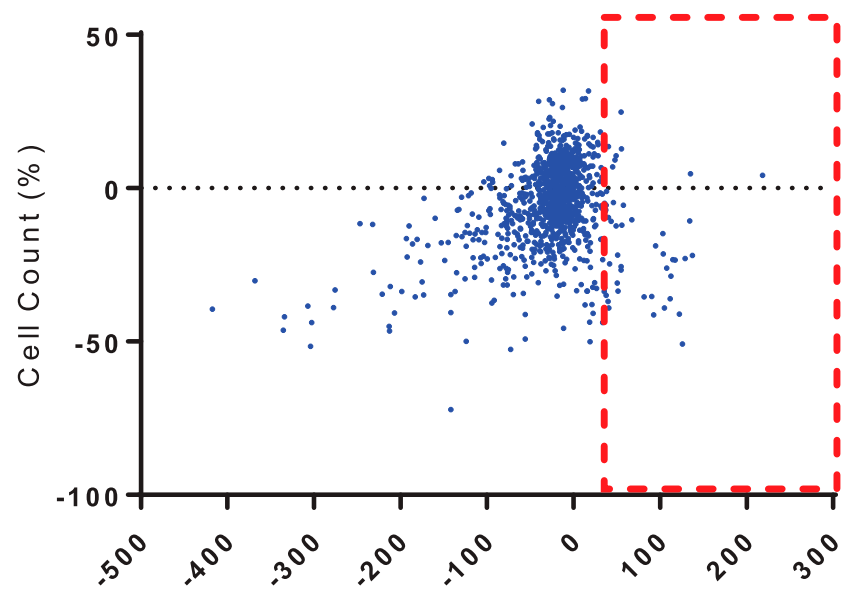

Max-response (\%)

C

G SK $3-\beta$ inhibitors

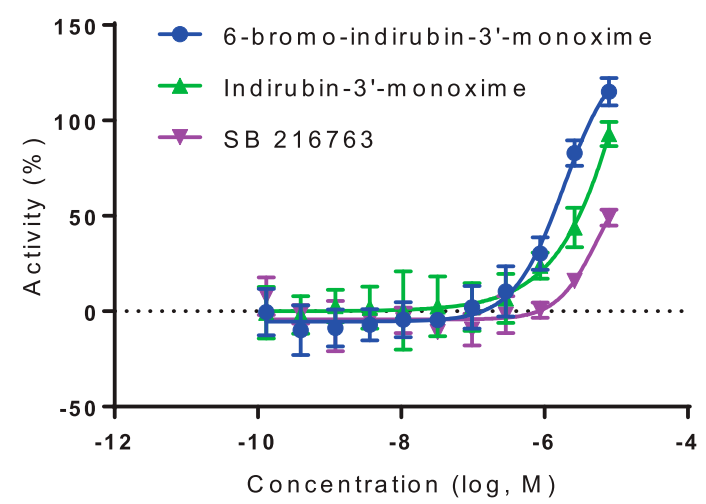

E

Calcium modulators

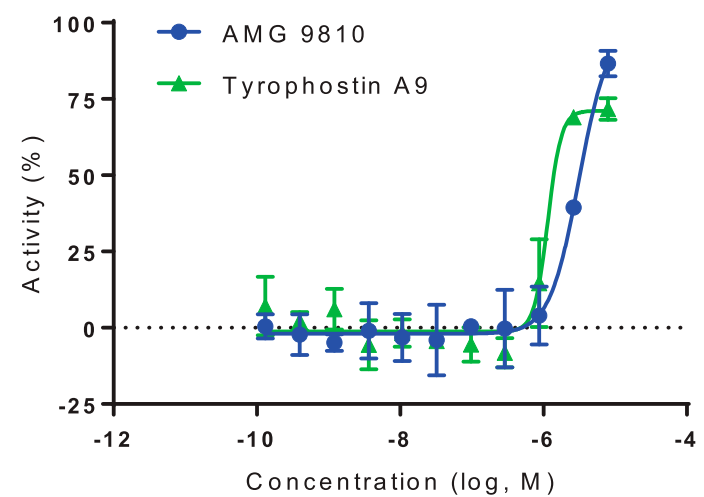

B

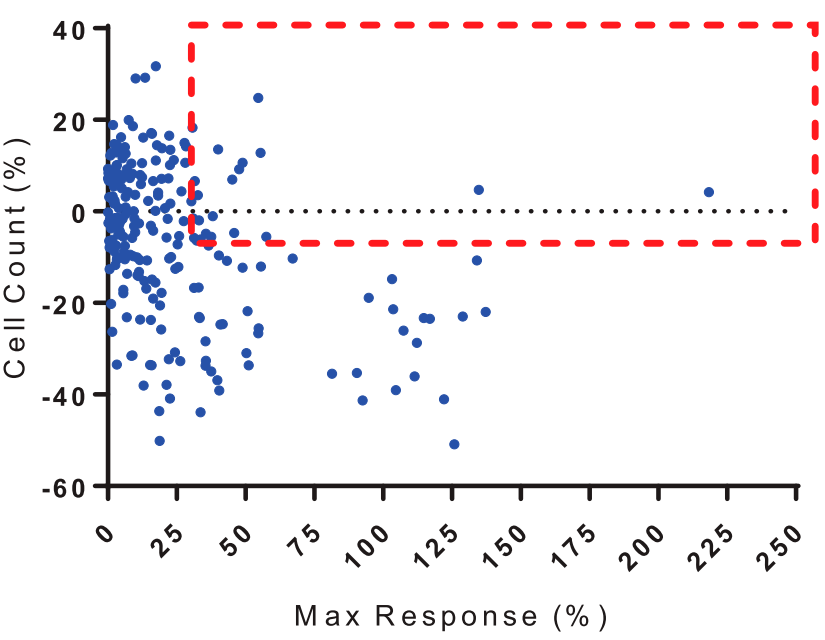

D

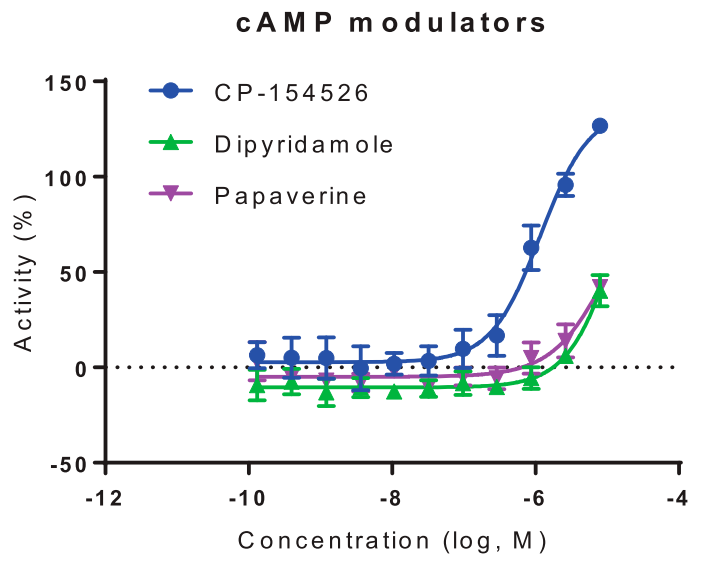

F

Receptor Tyrosine Kinase in hibitors

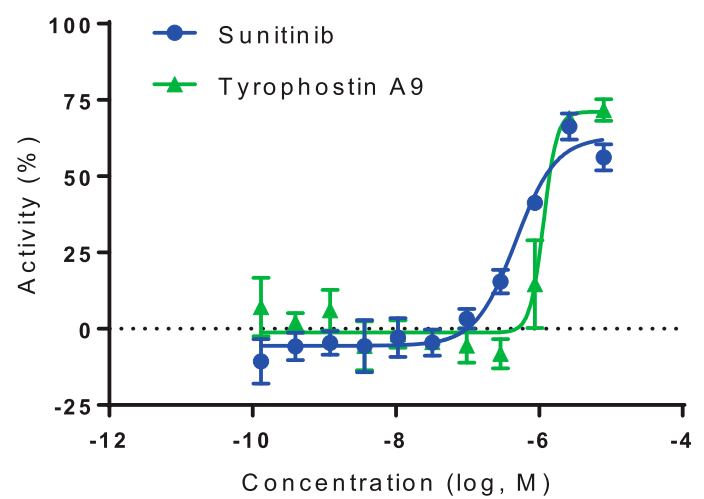

Fig. 4. Single-concentration LOPAC screening in 384-well plates. (A) Distribution of single-concentration compound-treated wells comparing the maximum response to cell count. A positive maximum response indicates a decrease in accumulated BODIPY-SM. Data were normalized to WT and NPA vehicle-treated controls ( $n=3$ interplate replicates). The red box indicates maximum responses above zero. (B) Selected wells for only maximum responses above zero. The red box indicates selected hits with a maximum response above 30\% and a reduction in cell count no more than $5 \%$. (C) Concentration-response curves for three GSK3- $\beta$ inhibitors. (D) Concentration-response curves for three cAMP modulators. (E) Concentration-response curves for two calcium modulators. (F) Concentration-response curves for two receptor tyrosine kinase (RTK) modulators. Data from (C-F) are averaged from three interplate replicates each and from three independent experiments. 
TABLE 1

Hits from secondary follow-up screen with $\mathrm{IC}_{50}$, efficacy, and $C_{\max }$ values

Table 1 shows the compound name, FDA status, the compound target, its reported $C_{\max }$ (if any), the experimentally determined $\mathrm{EC}_{50}$, the experimentally determined maximum response with S.D., and the reference for the $C_{\max }$.

\begin{tabular}{|c|c|c|c|c|c|c|}
\hline Compound Name & $\begin{array}{c}\text { FDA- } \\
\text { Approved }\end{array}$ & Target & $\begin{array}{l}C_{\max } \\
(\mu \mathrm{M})\end{array}$ & $\underset{(\mu \mathrm{M})}{\text { Average } \mathrm{EC}_{50}}$ & $\begin{array}{l}\text { Maximum } \\
\text { Response }\end{array}$ & Reference \\
\hline JFD00244 & No & SIRT2 inhibitor & N.D. & 7.5 & $154 \pm 5.6$ & \\
\hline $\begin{array}{l}\text { 6-Bromo-indirubin-3' - } \\
\text { monoxime }\end{array}$ & No & GSK3- $\beta$ inhibitor & N.D. & 1.9 & $115 \pm 7.3$ & \\
\hline Indirubin-3'-monoxime & No & $\begin{array}{l}\text { GSK3- } \beta \text { inhibitor, CDk5/1, 5- } \\
\text { lipoxygenase }\end{array}$ & N.D. & 42 & $92.9 \pm 10$ & \\
\hline SB 216763 & No & GSK3- $\alpha, \beta$ inhibitor & N.D. & 5.8 & $49.1 \pm 4.2$ & \\
\hline Staurosporine & No & PKC inhibitor & 0.035 & 0.74 & $65.6 \pm 6.1$ & Edelman et al., 2007 \\
\hline CP-154526 & No & CRF1 antagonist & N.D. & 1.2 & $127 \pm 3.8$ & \\
\hline Papaverine & Yes & PDE4/10 inhibitor, increased cAMP & 0.16 & 11 & $42 \pm 1.5$ & Kiessling et al., 2013 \\
\hline Dipyridamole & Yes & $\begin{array}{l}\text { PDE } 4 / 10 \text { and adenosine deaminase } \\
\text { inhibitor, increased cAMP }\end{array}$ & 7.4 & 16 & $40.2 \pm 8.2$ & Offman et al., 2013 \\
\hline Tyrophostin-A9 & No & PDGFR and CRAC inhibitor & N.D. & 1.1 & $71.7 \pm 3.6$ & \\
\hline Sunitinib & Yes & PDGFR/VEGFR inhibitor & 0.095 & 0.47 & $66.3 \pm 4.3$ & Kim et al., 2009 \\
\hline AMG 9810 & No & $\begin{array}{c}\text { Competitive vanilloid TRPV1 receptor } \\
\text { antagonist }\end{array}$ & N.D. & 3.1 & $86.6 \pm 4.2$ & \\
\hline $\begin{array}{l}S \text {-(p-Azidophenacyl) } \\
\text { glutathione }\end{array}$ & No & antioxidant & N.D. & 2.3 & $54.5 \pm 4.4$ & \\
\hline SB 242084 & No & 5-HT2c receptor inhibitor & N.D. & $\sim 3.0$ & $57.7 \pm 4.2$ & \\
\hline Diphenyleneiodonium & No & $\begin{array}{l}\text { Nitric oxide synthetase inhibitor, } \\
\text { NADPH oxidase inhibitor }\end{array}$ & N.D. & 0.0082 & $45.5 \pm 6.6$ & \\
\hline Cl-IB-MECA & No & A3 adenosine receptor agonist & 0.35 & 9.2 & $38.7 \pm 10$ & $\begin{array}{l}\text { van Troostenburg } \\
\text { et al., } 2004\end{array}$ \\
\hline Rotenone & No & Mitochondrial uncoupling & N.D. & 0.13 & $44.2 \pm 5.5$ & \\
\hline Niclosamide & Yes & Mitochondrial uncoupling & 0.0018 & $\sim 0.75$ & $90.6 \pm 3.4$ & Burock et al., 2018 \\
\hline
\end{tabular}

N.D., not determined; PKC, protein kinase C. SIRT2 - NAD-dependent deacetylase sirtuin 2. CDk5/1 - cyclin-dependent kinase 5/1. CRF1 - Corticotropin-releasing hormone

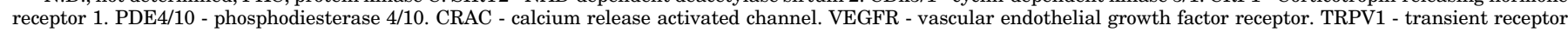
potential cation channel subfamily V member 1 . 5-HT2c - 5-hydroxytryptamine receptor 2C.

we first scanned a 384-well plate treated with $\mathrm{M} \beta \mathrm{CD}$ in concentration response on the Mirrorball and subsequently washed the plate to get rid of the background signal for imaging on the IN Cell (Supplemental Fig. 8). The results from the Mirrorball and IN Cell are comparable. To further compare the traditional cholesterol staining assay with the Mirrorball PFO* assay, we outlined the protocols in Supplemental Table 2 that indicate significant time savings.

Detecting Flavivirus Infection Using a Minimal-Wash Viral Titer Colony Assay. Lastly, we adapted the Mirrorball assay system to detect flavivirus infections in cells using a viral titer assay (Lee et al., 2019). We outlined the protocol for a traditional viral titer assay and our Mirrorball protocol in Supplemental Fig. 3 that indicates significant time saving with the Mirrorball assay. The single addition of a fluorescent protein-conjugated Envelope antibody against ZIKV and DENV was used to detect the viral colonies in monolayer Vero cells with methyl cellulose overlay. Although wash steps were used after fixation and permeabilization, the plate was not washed after the addition of the fluorescently labeled antibody. We employed a viral dilution protocol as a proof of concept in which lower concentrations of virus produce fewer colonies (Fig. 6). The results indicated that this Mirrorball assay using antibody-conjugated fluorescence staining can be used for detection of viral infections in cells.

\section{Discussion}

In this work, we have developed methods to detect cellular fluorescence staining in a no- or minimal-wash, high-density microplate format using the Mirrorball fluorescence cytometer. We have demonstrated the ability to detect both fluorescent staining of fluorescent dye-conjugated lipids and proteins in primary fibroblasts from patients with NPA and iPSC-derived NPC1 neural stem cells, respectively. The NPA BODIPY-SM accumulation assay was accomplished without any plate washing whatsoever in a true no-wash format. The NPC1 NSC PFO* cholesterol accumulation assay had one evacuation step. We also showcased the broad applications of this technique for no-wash cell-based assays through the detection of ZIKV and DENV in a viral titer assay using fluorescently labeled antibodies. Although there were some evacuation steps, these assays were developed without the need for washing and thus were able to minimize the variability introduced by wash steps.

This platform is a useful tool for phenotypic drug screening because of its minimal plate processing steps, which effectively increases assay robustness while minimizing well-towell variations. It also decreases data collection time and creates small data files. These Mirrorball assays can be further enhanced with the use of robotic arms and plate stacker systems for HTS. The system is particularly adaptable to various fluorescence assays, with five distinct fluorescence channels, adjustable laser power, and adjustable photomultiplier tube voltage settings to customize the system for different fluorescent combinations and intensities. The selectable acquisition triggers and sensitivity settings allow for data acquisition once the fluorescence intensity value is greater than a certain number of standard deviations above background, resulting in preselection of the relevant results from the entire well, rapid data calculation, and small output data file. Although the resolution is limited, the entire well can be scanned to provide a clear view of the cell distribution in wells and fluorescence staining in cells.

LSDs have characteristic accumulation of one or more substrates within the lysosome as a result of gene mutations 
A

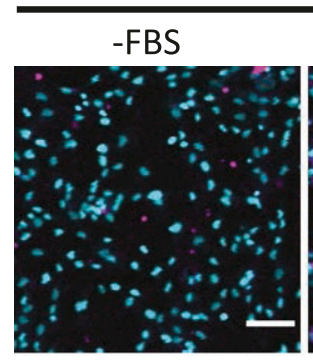

$+\mathrm{FBS}$

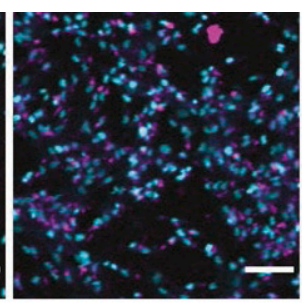

B
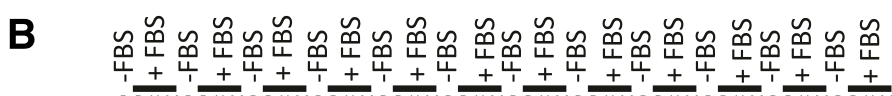

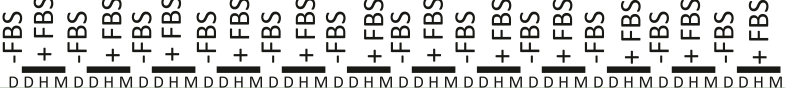

$300 \mu \mathrm{M}$

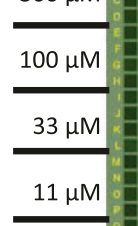

$3.7 \mu \mathrm{M}$

$1.2 \mu \mathrm{M}$

$0.41 \mu \mathrm{M}$

$0.14 \mu \mathrm{M}$

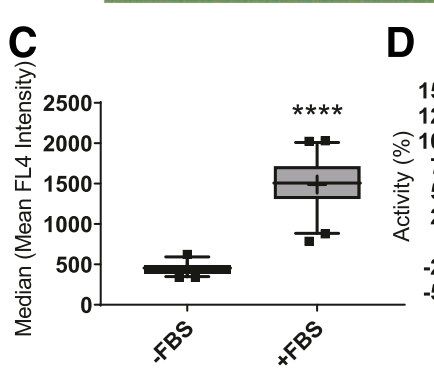

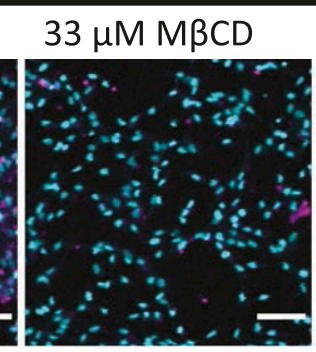

NPC1 HT268A GM03123

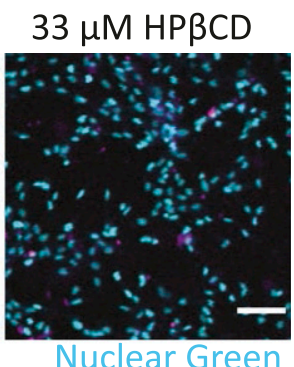

Nuclear Green PFO*

\section{D - DMSO}

$H-H P \beta C D$

$M-M \beta C D$
Fig. 5. Development of a minimal-wash $\mathrm{PFO}^{*}$ cholesterol assay for NPC1 disease using NSCs. (A) Background-subtracted and cropped TIFF images of NSCs stained with Nuclear Green LCS1 (cyan) and iFluor-647-PFO (magenta). Scale bar, $100 \mu \mathrm{m}$. (B) Image of the plate heatmap demonstrating median (mean FL2 intensity) of NPC1 NSCs treated with and without FBS and DMSO, M $\beta C D$, or $\mathrm{HP} \beta C D$. (C) Comparison of NPC1 NSC cholesterol accumulation in the absence (black) or presence (gray) of FBS in the growth culture media. Data shown as box and whisker plots, with whiskers showing $5 \%$ and $95 \%$ percentiles; + indicates mean. $* * * * P<0.0001$ using unpaired $t$ test with Welch's correction. (D) Concentration-response curve for $\mathrm{M} \beta \mathrm{CD}$ (blue squares and curve) or $\mathrm{HP} \beta \mathrm{CD}$ (red circles and curve) ( $n=3$ independent experiments). (E) Cell counts for NSCs treated with $\mathrm{M} \beta \mathrm{CD}$ (blue squares) or HP $\beta \mathrm{CD}$ (red circles) ( $n=3$ independent experiments). Data normalized to NPC1 NSCs treated with and without FBS. Curves represent nonlinear regression curve fit with variable slope. of lysosomal proteins that disrupt functions needed for substrate clearance. As such, the cellular processes that rely on good lysosomal health and autophagic flux can be dysregulated, leading to a multitude of pathologic effects. In NPC1, research has shown that autophagic flux is impaired because of the burden of cholesterol accumulation in the lysosome, and activation of AMP-activated protein kinase with $\mathrm{M} \beta \mathrm{CD}$ alleviates the NPC1 disease phenotype (Dai et al., 2017). Another study has shown that HP $\beta C D$ interacts with the lysosomal membrane-associate protein 1 to facilitate cholesterol egress from patient skin fibroblasts (Singhal et al., 2018). Although not all LSDs present with the same impairments in autophagy, dysregulated autophagy can be considered a hallmark and common mechanism of LSDs (Seranova et al., 2017).

In this study, we tested the Mirrorball system for compatibility with phenotypic fluorescent cell-based screening methods. $\delta$-Tocopherol, a component of vitamin $\mathrm{E}$, has been reported to alleviate sphingomyelin accumulation in Niemann-Pick disease type $\mathrm{C}$ and other lysosomal storage diseases, including NPA (Xu et al., 2012). In our no-wash Mirrorball assay, the observed $\mathrm{EC}_{50}$ in NPA fibroblasts was $16.1 \mu \mathrm{M}$, which corresponds well to the previously reported
$\mathrm{EC}_{50}$ of $13.2 \mu \mathrm{M}$. Similar $\mathrm{EC}_{50}$ values for $\delta$-tocopherol were observed in NPA NSCs (Long et al., 2016). We also found that the addition of $\mathrm{HP} \beta \mathrm{CD}$ in combination with $\delta$-tocopherol increased the efficacy of the treatment.

Cyclodextrin has also been under evaluation for the treatment of NPC1 since 2008 after it exhibited beneficial treatment effects in an $\mathrm{Npc1}^{-/-}$mouse model (Davidson et al., 2009; Liu et al., 2009). Since then, it has made its way successfully into clinical trials (Matsuo et al., 2013; Ory et al., 2017). As of now, NPC1 does not have an FDA-approved treatment. Therefore, the clinical evaluation of cyclodextrin in patients is extremely important to those suffering from the disease (clinicaltrials.gov identifier: NCT02534844). HP $\beta$ CD was shown to slow the disease progression in the phase $1-2$ a clinical study (Ory et al., 2017). However, in the phase 2b/3 clinical trial, early reports did not demonstrate statistically significant differences between cohorts. Thus, the development of $\mathrm{HP} \beta C D$ continues.

Here, we used NPC1 NSCs to evaluate the Mirrorball's capacity for detecting cholesterol staining with the fluorescent dye-conjugated cholesterol sensing protein $\mathrm{PFO}$ with minimal plate washing. Previously reported $\mathrm{EC}_{50}$ values for cyclodextrins 
A
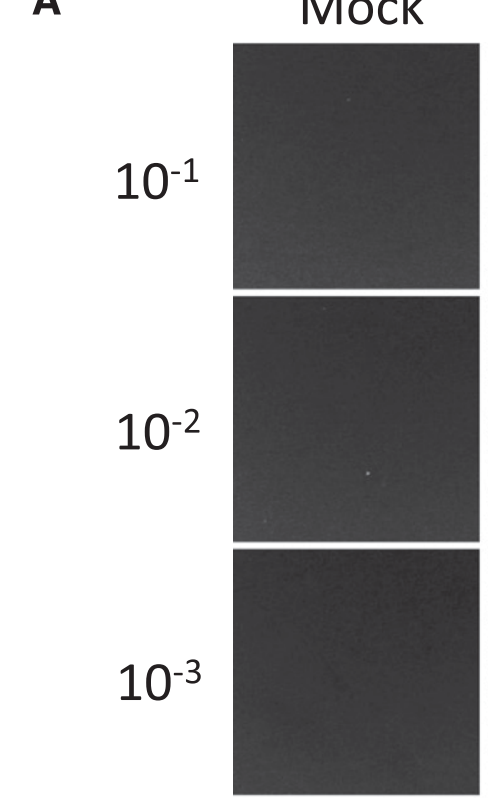

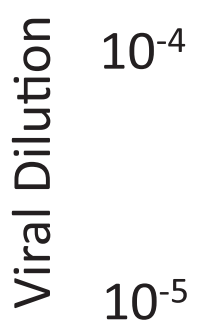
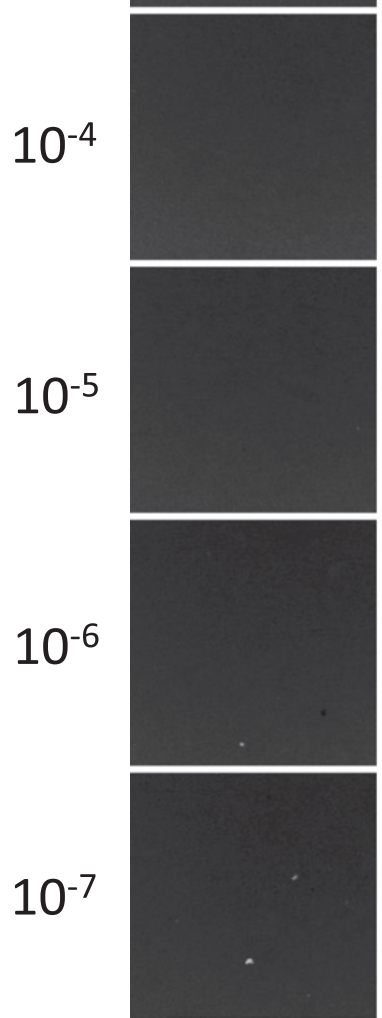

$10^{-8}$
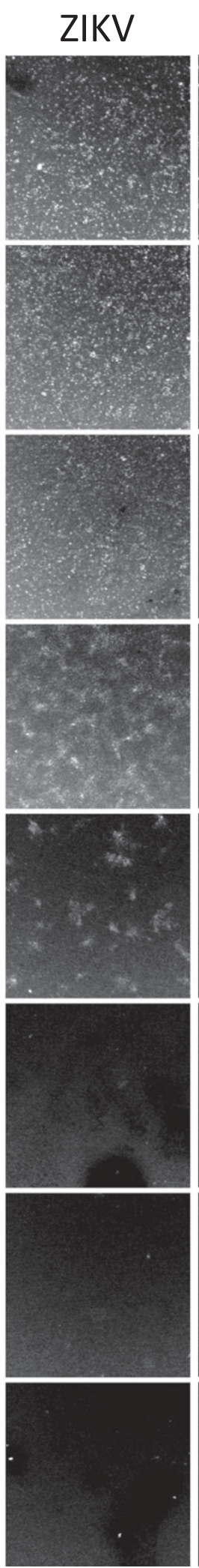

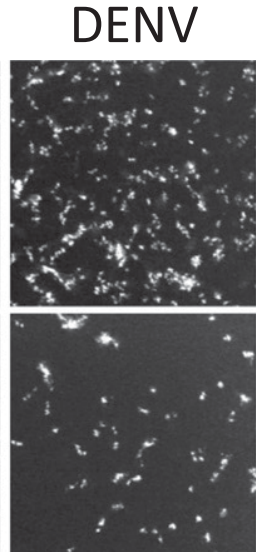

B

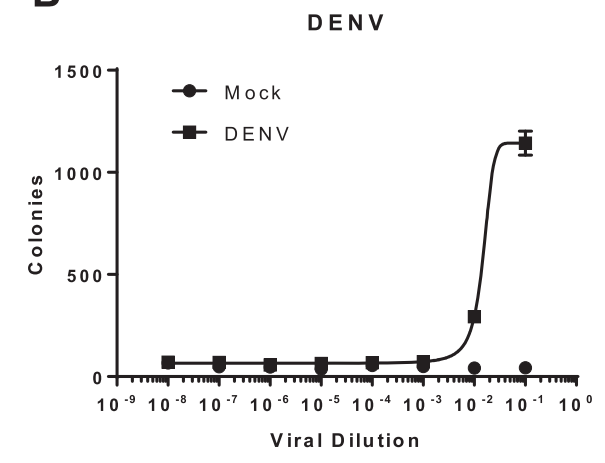

C
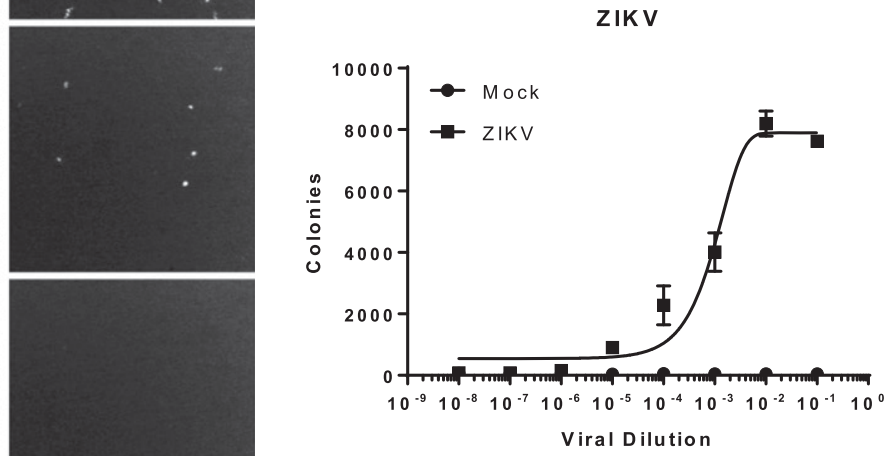

Fig. 6. Viral titer assay for ZIKV and DENV. (A) Fields of view from wells of a 96-well plate for mock, ZIKV, and DENV viral titer assay. Each row represents a representative well, arranged from low to high dilution of virus. (B) DENV colony counts ( $n=3$ wells). (C) ZIKV colony counts $(n=3$ wells). Curves were generated using nonlinear regression. 
$\mathrm{M} \beta \mathrm{CD}$ and $\mathrm{HP} \beta \mathrm{CD}$ were 14.3 and $26.3 \mu \mathrm{M}$ in the filipin cholesterol staining assay, respectively (Yu et al., 2014). Although our assay provided similar $\mathrm{EC}_{50}$ results for these two cyclodextrins, we do observe a lower $\mathrm{EC}_{50}$ for $\mathrm{M} \beta \mathrm{CD}$ than $\mathrm{HP} \beta \mathrm{CD}$, at 3.13 and $8.17 \mu \mathrm{M}$, respectively.

In addition to using known compounds to validate the Mirrorball assays, we also screened compound collections to identify new hits that reduced the lipid accumulation phenotype in the cells from patients with NPA. From our screen, GSK3- $\beta$ inhibitors were identified as potential molecules that may reduce sphingomyelin accumulation in fibroblasts from patients with NPA. GSK3- $\beta$ is a kinase that is involved in many cellular functions, including energy metabolism and neuronal cell development (Mancinelli et al., 2017). Given that GSK3- $\beta$ regulates the mammalian target of rapamycin (mTOR) by phosphorylating Raptor within the mTOR complex 1 , it is possible that the GSK3- $\beta$ inhibitors may reduce the accumulation of sphingomyelin by stimulating autophagic flux (Azoulay-Alfaguter et al., 2015). There are a number of clinical trials exploring other GSK3- $\beta$ inhibitors for central nervous system diseases and cancer (Eldar-Finkelman and Martinez, 2011; Mancinelli et al., 2017).

Both papaverine and dipyridamole inhibit phosphodiesterase 4 and 10, resulting in increased cAMP concentrations in the cell (Kiessling et al., 2013; Offman et al., 2013). Increases in cAMP result in direct activation of protein kinase A (PKA) in distinct subcellular compartments within the cell facilitated by the scaffolding PKA anchoring protein (Gorshkov and Zhang, 2014; Gorshkov et al., 2017). Normally, PKA is considered a negative regulator of autophagy via its phosphorylation of 5' AMP-activated protein kinase inhibitory sites, mTOR, and microtubule-associated proteins 1A/1B light chain 3B (LC3) (Djouder et al., 2010; Chen and Klionsky, 2011). Interestingly, PKA is a negative upstream regulator of GSK3- $\beta$ through phosphorylation of serine 21 and serine 9 (Fang et al., 2000) and thus could lead to similar effects on autophagy flux. Additionally, cAMP has been reported to be associated with increased autophagy induction (Ugland et al., 2011).

Two platelet-derived growth factor receptor (PDGFR) inhibitors, tyrophostin A9 and sunitinib, reduced accumulation in NPA fibroblasts, with fairly potent $\mathrm{EC}_{50}$ values of 1.1 and 0.47 $\mu \mathrm{M}$, respectively. Sunitinib was approved by the FDA in 2006 for renal cell carcinoma and gastrointestinal stromal tumor. PDGF signaling inhibition has been reported to induce autophagy in malignant glioma cells (Takeuchi et al., 2004). Sunitinib also promoted autophagy in an human immunodeficiency virus 1 Tat neurodegenerative mouse model (Fields et al., 2017). Increased LC3-II and reduced p62 protein, indicators of autophagic flux, were observed in neuronal cultures from the transgenic Tat mice, and in vivo sunitinib normalized the levels of LC3-II, p62, and endophilin B1 (Fields et al., 2017). Future work will validate these and other compounds for accumulation of sphingomyelin and explore related mechanisms of action.

To alleviate the existing technical bottlenecks in the early drug discovery workflow, researchers must adopt new assay technologies, including imaging platforms and automation solutions, with the goal of enhancing screening capacity and efficiency. The use of good disease models coupled with the appropriate screening assays provides a strong foundation for accelerating the drug development process. Simplifying phenotypic screening assays that bear known disease phenotypes can increase assay robustness and fulfill the need for primary screening solutions. Therefore, the Mirrorball assay technology tested in this study can be broadly applied to highcontent and phenotypic assays to improve the efficiency and quality of HTS.

Data Availability. Data are available upon request.

\section{Acknowledgments}

Thanks to the team from TTP Labtech for training and helping to optimize the Mirrorball settings for our assays. Thanks to Charles Yeh for initial testing of the Mirrorball.

\section{Authorship Contributions}

Participated in research design: Gorshkov, Zheng.

Conducted experiments: Gorshkov, Pradhan, Xu, Yang, Lee, Chen, Zheng.

Performed data analysis: Gorshkov, Shen

Wrote or contributed to the writing of the manuscript: Gorshkov, Pradhan, Xu, Lee, Shen, Zheng.

\section{References}

Azoulay-Alfaguter I, Elya R, Avrahami L, Katz A, and Eldar-Finkelman H (2015) Combined regulation of mTORC 1 and lysosomal acidification by GSK-3 suppresses autophagy and contributes to cancer cell growth. Oncogene 34:4613-4623.

Balmaseda A, Stettler K, Medialdea-Carrera R, Collado D, Jin X, Zambrana JV, Jaconi S, Cameroni E, Saborio S, Rovida F, et al. (2017) Antibody-based assay discriminates Zika virus infection from other flaviviruses. Proc Natl Acad Sci USA 114:8384-8389.

Burock S, Daum S, Keilholz U, Neumann K, Walther W, and Stein U (2018) Phase II trial to investigate the safety and efficacy of orally applied niclosamide in patients with metachronous or sychronous metastases of a colorectal cancer progressing after therapy: the NIKOLO trial. BMC Cancer 18:297.

Chen Y and Klionsky DJ (2011) The regulation of autophagy - unanswered questions. $J$ Cell Sci 124:161-170.

Dai S, Dulcey AE, Hu X, Wassif CA, Porter FD, Austin CP, Ory DS, Marugan J, and Zheng W (2017) Methyl- $\beta$-cyclodextrin restores impaired autophagy flux in Niemann-Pick C1-deficient cells through activation of AMPK. Autophagy 13: 1435-1451.

Davidson CD, Ali NF, Micsenyi MC, Stephney G, Renault S, Dobrenis K, Ory DS, Vanier MT, and Walkley SU (2009) Chronic cyclodextrin treatment of murine Niemann-Pick C disease ameliorates neuronal cholesterol and glycosphingolipid storage and disease progression. PLoS One 4:e6951.

Djouder N, Tuerk RD, Suter M, Salvioni P, Thali RF, Scholz R, Vaahtomeri K, Auchli Y, Rechsteiner H, Brunisholz RA, et al. (2010) PKA phosphorylates and inactivates AMPKalpha to promote efficient lipolysis. EMBO $J$ 29:469-481.

Edelman M, Bauer K, Wu S, Smith R, Bisacia S, and Dancey J (2007) Phase I and pharmacokinetic study of 7-hydroxystaurosporine and carboplatin in advanced solid tumors. Clin Cancer Res 13:2667-2674.

Eldar-Finkelman H and Martinez A (2011) GSK-3 inhibitors: preclinical and clinical focus on CNS. Front Mol Neurosci 4:32.

England E, Newton P, Neal F, Kitching L, Colley C, and Rossant CJ (2015) Application of the mirrorball high-sensitivity cytometer to multiplexed assays for antibody drug discovery. J Biomol Screen 20:536-544.

Fang X, Yu SX, Lu Y, Bast RC Jr., Woodgett JR, and Mills GB (2000) Phosphorylation and inactivation of glycogen synthase kinase 3 by protein kinase A. Proc Nat Acad Sci USA 97:11960-11965.

Fields JA, Metcalf J, Overk C, Adame A, Spencer B, Wrasidlo W, Florio J, Rockenstein E, He JJ, and Masliah E (2017) The anticancer drug sunitinib promotes autophagyand protects from neurotoxicity in an HIV-1 Tat model of neurodegeneration. J Neurovirol 23:290-303.

Gorshkov K, Mehta S, Ramamurthy S, Ronnett GV, Zhou F-Q, and Zhang J (2017) AKAP-mediated feedback control of cAMP gradients in developing hippocampal neurons. Nat Chem Biol 13:425-431.

Gorshkov K and Zhang J (2014) Visualization of cyclic nucleotide dynamics in neurons. Front Cell Neurosci 8:395.

Johnson BB, Breña M, Anguita J, and Heuck AP (2017) Mechanistic insights into the cholesterol-dependent binding of perfringolysin O-based probes and cell membranes. Sci Rep 7:13793.

Kiessling AH, Romasku D, Beiras-Fernandez A, Ferreirós N, Labocha S, Moritz A and Rastan AJ (2013) Pharmacokinetics of intraluminally administered serum papaverine for spasm prophylaxis of the internal mammary artery. Heart Surg Forum 16:E266-E270.

Kim A, Balis FM, and Widemann BC (2009) Sorafenib and sunitinib. Oncologist 14: 800-805.

Lee EM, Titus SA, Xu M, Tang H, and Zheng W (2019) High-throughput Zika viral titer assay for rapid screening of antiviral drugs. Assay Drug Dev Technol 17: 128-139.

Liu B, Turley SD, Burns DK, Miller AM, Repa JJ, and Dietschy JM (2009) Reversal of defective lysosomal transport in NPC disease ameliorates liver dysfunction and neurodegeneration in the npc1-/- mouse. Proc Natl Acad Sci USA 106:2377-2382. 
Long Y, Xu M, Li R, Dai S, Beers J, Chen G, Soheilian F, Baxa U, Wang M, Marugan JJ, et al. (2016) Induced pluripotent stem cells for disease modeling and evaluation of therapeutics for Niemann-Pick disease type A. Stem Cells Transl Med 5: 1644-1655.

Maekawa M, Yang Y, and Fairn GD (2016) Perfringolysin O theta toxin as a tool to monitor the distribution and inhomogeneity of cholesterol in cellular membranes. Toxins (Basel) 8:67.

Mancinelli R, Carpino G, Petrungaro S, Mammola CL, Tomaipitinca L, Filippini A, Facchiano A, Ziparo E, and Giampietri C (2017) Multifaceted roles of GSK-3 in cancer and autophagy-related diseases. Oxid Med Cell Longev 2017:4629495.

Matsuo M, Togawa M, Hirabaru K, Mochinaga S, Narita A, Adachi M, Egashira M, Irie T, and Ohno K (2013) Effects of cyclodextrin in two patients with NiemannPick Type C disease. Mol Genet Metab 108:76-81.

Nishiyama K, Suzuki H, Maruyama M, Yoshihara T, and Ohta H (2017) Genetic deletion of GPR52 enhances the locomotor-stimulating effect of an adenosine $\mathrm{A}_{2 \mathrm{~A}}$ receptor antagonist in mice: a potential role of GPR52 in the function of striatopallidal neurons. Brain Res 1670:24-31.

Offman E, Schobelock MJ, Brickl R, VanderMaelen CP, Ehrlich J, and Eisert W (2013) Pharmacokinetics and pharmacodynamics of the antiplatelet combination aspirin (acetylsalicylic acid) plus extended-release dipyridamole are not altered by coadministration with the potent CYP2C19 inhibitor omeprazole. Am J Cardiovasc Drugs 13:113-120.

Ory DS, Ottinger EA, Farhat NY, King KA, Jiang X, Weissfeld L, Berry-Kravis E, Davidson CD, Bianconi S, Keener LA, et al. (2017) Intrathecal 2-hydroxypropyl$\beta$-cyclodextrin decreases neurological disease progression in Niemann-Pick disease, type C1: a non-randomised, open-label, phase 1-2 trial. Lancet 390:1758-1768.

Priest BT and Erdemli G (2014) Phenotypic screening in the 21st century. Front Pharmacol 5:264.

Rasmussen L, Tigabu B, White EL, Bostwick R, Tower N, Bukreyev A, Rockx B, LeDuc JW, and Noah JW (2015) Adapting high-throughput screening methods and assays for biocontainment laboratories. Assay Drug Dev Technol 13:44-54.

Schuchman EH and Desnick RJ (2017) Types A and B Niemann-Pick disease. Mol Genet Metab 120:27-33.

Seranova E, Connolly KJ, Zatyka M, Rosenstock TR, Barrett T, Tuxworth RI, and Sarkar S (2017) Dysregulation of autophagy as a common mechanism in lysosomal storage diseases. Essays Biochem 61:733-749.
Singhal A, Szente L, Hildreth JEK, and Song B (2018) Hydroxypropyl-beta and -gamma cyclodextrins rescue cholesterol accumulation in Niemann-Pick C1 mutant cell via lysosome-associated membrane protein 1. Cell Death Dis $\mathbf{9}$ 1019 .

Takeuchi H, Kanzawa T, Kondo Y, and Kondo S (2004) Inhibition of platelet-derived growth factor signalling induces autophagy in malignant glioma cells. $\mathrm{Br} \mathrm{J}$ Cancer 90:1069-1075.

Ugland H, Naderi S, Brech A, Collas P, and Blomhoff HK (2011) cAMP induces autophagy via a novel pathway involving ERK, cyclin E and Beclin 1. Autophagy 7 1199-1211.

van Troostenburg AR, Clark EV, Carey WD, Warrington SJ, Kerns WD, Cohn I, Silverman MH, Bar-Yehuda S, Fong KL, and Fishman P (2004) Tolerability, pharmacokinetics and concentration-dependent hemodynamic effects of ora $\mathrm{CF} 101$, an $\mathrm{A} 3$ adenosine receptor agonist, in healthy young men. Int $\mathrm{J}$ Clin Pharmacol Ther 42:534-542.

Xu M, Liu K, Swaroop M, Porter FD, Sidhu R, Firnkes S, Ory DS, Marugan JJ, Xiao $J$, Southall N, et al. (2012) $\delta$-Tocopherol reduces lipid accumulation in NiemannPick type $\mathrm{C} 1$ and Wolman cholesterol storage disorders [published correction appears in J Biol Chem (2013) 288:296]. J Biol Chem 287:39349-39360.

Yang S, Xu M, Lee EM, Gorshkov K, Shiryaev SA, He S, Sun W, Cheng Y-S, Hu X, Tharappel AM, et al. (2018) Emetine inhibits Zika and Ebola virus infections through two molecular mechanisms: inhibiting viral replication and decreasing viral entry. Cell Discov 4:31.

Yu D, Swaroop M, Wang M, Baxa U, Yang R, Yan Y, Coksaygan T, DeTolla L, Marugan JJ, Austin CP, et al. (2014) Niemann-pick disease type C: induced pluripotent stem cell-derived neuronal cells for modeling neural disease and evaluating drug efficacy. J Biomol Screen 19:1164-1173.

Zanella F, Lorens JB, and Link W (2010) High content screening: seeing is believing. Trends Biotechnol 28:237-245.

Address correspondence to: Dr. Kirill Gorshkov, NCATS/NIH, 9800 Medical Center Drive, MSC: 3375, Bethesda, MD 20892 E-mail: kirill.gorshkov@nih.gov; or Dr. Wei Zheng, NCATS/NIH, 9800 Medical Center Drive MSC: 3375, Bethesda, MD 20892. E-mail: wzheng@mail.nih.gov 